Sodtke, Diana; Armbruster, Meinrad M.

\title{
ELTERN-AG - Die niedrigschwellige Elternschule für die frühe Kindheit
}

Praxis der Kinderpsychologie und Kinderpsychiatrie 56 (2007) 8, S. 707-720

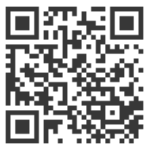

Quellenangabe/ Reference:

Sodtke, Diana; Armbruster, Meinrad M.: ELTERN-AG - Die niedrigschwellige Elternschule für die frühe Kindheit - In: Praxis der Kinderpsychologie und Kinderpsychiatrie 56 (2007) 8, S. 707-720 - URN: urn:nbn:de:0111-opus-30619 - DOI: 10.25656/01:3061

in Kooperation mit / in cooperation with:

\section{Vandenhoeck \& Ruprecht}

http://www.v-r.de

\section{Nutzungsbedingungen}

Gewährt wird ein nicht exklusives, nicht übertragbares, persönliches und beschränktes Recht auf Nutzung dieses Dokuments. Dieses Dokument ist ausschließlich für den persönlichen, nicht-kommerziellen Gebrauch bestimmt. Die Nutzung stellt keine Übertragung des Eigentumsrechts an diesem Dokument dar und gilt vorbehaltlich der folgenden Einschränkungen: Auf sämtlichen Kopien dieses Dokuments müssen alle Urheberrechtshinweise und sonstigen Hinweise auf gesetzlichen Schutz beibehalten werden. Sie dürfen dieses Dokument nicht in irgendeiner Weise abändern, noch dürfen Sie dieses Dokument für öffentliche oder kommerzielle Zwecke vervielfältigen, öffentlich ausstellen, aufführen, vertreiben oder anderweitig nutzen.

Mit der Verwendung dieses Dokuments erkennen Sie die Nutzungsbedingungen an.

\section{Terms of use}

We grant a non-exclusive, non-transferable, individual and limited right to using this document.

This document is solely intended for your personal, non-commercial use. Use of this document does not include any transfer of property rights and it is conditional to the following limitations: All of the copies of this documents must retain all copyright information and other information regarding legal protection. You are not allowed to alter this document in any way, to copy it for public or commercial purposes, to exhibit the document in public, to perform, distribute or otherwise use the document in public.

By using this particular document, you accept the above-stated conditions of use.

\section{Kontakt / Contact:}

\section{DeDOCS}

DIPF | Leibniz-Institut für Bildungsforschung und Bildungsinformation

Informationszentrum (IZ) Bildung

E-Mail: pedocs@dipf.de

Internet: www.pedocs.de

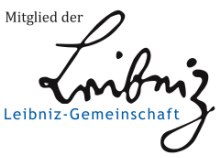




\title{
ELTERN-AG - \\ Die niedrigschwellige Elternschule für die frühe Kindheit
}

\author{
Diana Sodtke und Meinrad M. Armbruster
}

\begin{abstract}
Summary
ELTERN-AG - the low threshold school for parents for the early childhood

ELTERN-AG is a selective program of primary prevention. It is a low threshold program and establishes resources of the parents. The groups are homogeneous which means that all families have similar problems. It works with deprived parents from the moment when they plan to get a baby up to their child's school entry. The concept ELTERN-AG theoretically derives from the need-oriented psychological model set up by Epstein (1990) and Grawe (2004) and was modified by Armbruster (2006). It assumed, that deprived people are prevented from meeting their essential needs, so that they are not able to perceive and meet the children's basic needs adequately. ELTERN-AG aims in 20 sessions at the training of competences in early education and the strengthening of the parents' self-help potentials through empowerment. The results of the 3-year long scientific accompanying research show that parents that participated in a course see themselves strengthened when dealing with their children, assess in total the relationship to children more positively and are more confident when coping with problems which occur in connection with their children. During the course parents notice a change about themselves which is recognized by mentors as well as professional multipliers and is confirmed as positive change.
\end{abstract}

Prax. Kinderpsychol. Kinderpsychiat. 56/2007, 707-720

\section{Keywords}

Parental school - deprived clientele - empowerment approach - need-oriented psychological model

\section{Zusammenfassung}

ELTERN-AG ist ein primäres selektives Präventionsprogramm, charakterisiert durch die Qualitätsmerkmale Niedrigschwelligkeit, Ressourcenorientiertheit und homogene Gruppenzusammensetzung. Es arbeitet mit sozial benachteiligten Eltern von der Familienplanungsphase bis zum Schuleintritt der Kinder. Theoretisch beruht das ELTERN-AG-Konzept auf dem bedürfnispsychologischen Modell von Epstein $(1990,1993)$ und Grawe (2004), modifiziert von Armbruster (2006). Es geht davon aus, dass Menschen mit sozialer Benachteiligung an der Befriedigung ihrer essentiellen Bedürfnisse gehindert sind, so dass sie nicht in der Lage sind, die Grundbedürfnisse ihrer eigenen Kinder angemessen wahrzunehmen und zu befriedigen. Die ELTERN-AG zielt in 20 Sitzungen auf die Schulung der Früherziehungskompetenzen und die Stärkung der Selbsthilfepotentiale von Eltern durch Empowerment ab. Die Ergebnisse der dreijährigen wissenschaftlichen Begleitforschung zeigen, dass sich die teilneh- 
menden Eltern als gestärkt im Umgang mit ihren Kindern erleben, insgesamt die Beziehung zu ihren Kindern positiver einschätzen und sich sicherer im Umgang mit Problemen fühlen, die im Zusammenhang mit ihren Kinder auftreten. Der Wandel, den die Eltern während des Kurses an sich wahrnehmen, fällt sowohl den Mentoren als auch professionellen Multiplikatoren auf und wird als positive Veränderung bestätigt.

\section{Schlagwörter}

Elternschule - sozial benachteiligtes Klientel - Empowerment-Ansatz - bedürfnispsychologisches Modell

\section{Die Rolle der sozialen Herkunft}

„Deutschland missachtet den Anfang und die Anfänger,“ resümierte Andreas Schleicher am 16.9.2003 bei der Vorstellung der OECD Studie „Bildung auf einen Blick 2003“. Der Initiator der PISA-Studie sieht in der Vernachlässigung von Kindern aus einkommensschwachen und bildungsfernen Schichten einen Hauptgrund für das schlechte Abschneiden Deutschlands im internationalen Vergleich. PISAund IGLU-Studie werfen ein bezeichnendes Licht auf den Sachverhalt, wie erheblich der Einfluss der sozialen Herkunft der Kinder auf ihr schulisches Bildungs- und Leistungsniveau ist (vgl. Baumert, 2001; Bos et al., 2003). In der internationalen European Child Care and Education Study (Krumm et al., 1999) wird aufgezeigt, dass der Einfluss des Elternhauses in der frühen Kindheit mit über $50 \%$ die Wirkungen der öffentlichen Angebote wie Kinderkrippen und Kindertagesstätten deutlich übertrifft. Die internationalen Forschungsarbeiten belegen auf empirischer Basis, dass kompensatorische Förderung und Bildungsangebote im Elementar- und Grundschulalter essentiell für die gesamte weitere Entwicklung sind und Benachteiligungen auszugleichen vermögen (Fthenakis, 2003). Was dort versäumt wird, holen die Schüler meist in den höheren Klassenstufen meist nicht mehr auf. Nur $30 \%$ eines Jahrgangs fangen in Deutschland ein Studium an, in Finnland hingegen studieren $70 \%$, der OECD-Durchschnitt liegt bei $45 \%$.

Als primäre Bezugspersonen für die ersten Lebensjahre bedürfen gerade Eltern in defizitären Lebenslagen einer besonderen und spezifischen Unterstützung, damit ihre Kinder solide Grundlagen erhalten. Die Forschungsergebnisse zeigen übereinstimmend, dass die stabile seelische Verfassung der einzelnen Kinder, die sie in den Elternhäusern vermittelt bekommen die elementare Voraussetzung darstellt, damit sie Kindergärten und Schulen effektiv nutzen können. Wenn Eltern früh lernen, geeignete Kompetenzen zu erwerben und einzusetzen, die ihren Kindern angemessene altersentsprechende Entwicklungsschritte ermöglichen, erfahren sie sich als selbstwirksame Autoren der kindlichen Erfolge und werden mit jedem Erfolg weiter in ihrem positiven Erziehungsverhalten bekräftigt.

(C) Vandenhoeck \& Ruprecht GmbH \& Co. KG, Göttingen 2007 


\section{Bedürfnis- und entwicklungspsychologische Grundlagen}

ELTERN-AG ist ein Präventionsprogramm, das ausgehend von dem bedürfnispsychologischen Theorienmodell von Epstein $(1990,1993)$ und Grawe (2004) auf die Schulung der Früherziehungskompetenzen und die Stärkung der Selbsthilfepotentiale von Eltern durch Empowerment abzielt. Aufgrund von Bildungsferne, Armut und Migrationshintergrund sind Eltern mit sozialer Benachteiligung an der Befriedigung ihrer essentiellen Bedürfnisse gehindert, so dass sie nicht in der Lage sind, die Grundbedürfnisse ihrer eigenen Kinder angemessen wahrzunehmen und zu befriedigen.

Tab. 1: Psychische Grundbedürfnisse

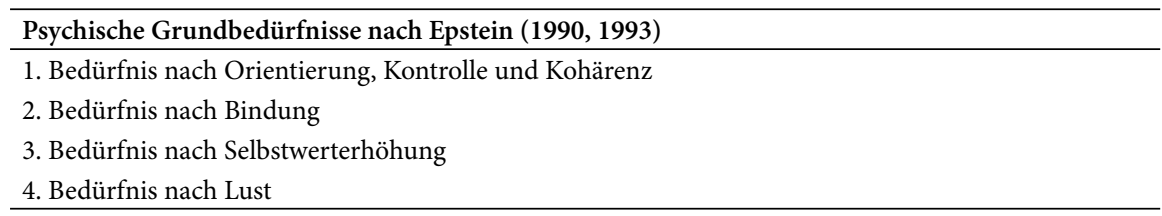

Kinder mit Risikobedingungen entwickeln in ihren ersten Lebensjahren häufig ein geringes Selbstbewusstsein, mangelndes Konzentrations- und Durchsetzungsvermögen, schwache Durchsetzungskraft, gelernte Hilflosigkeit und letztlich psychische Labilität, wenn die psychischen Grundbedürfnisse dauerhaft frustriert werden. Diese frühen vorsprachlichen Erfahrungen hinterlassen neurologisch ungünstige Bahnungen und wirken als funktionale Narben (Braun, 2006). Nach Grawe (2004) sind alle Menschen auf psychische Konsistenz ausgerichtet. Sie stellt das Streben nach einem übergeordneten Wohlbefinden dar, das auf einer Befriedigung der Grundbedürfnisse beruht. Bleiben ihre Bedürfnisse dauerhaft ungestillt, leiden sie an einem hohen Maß an Inkonsistenz, weil sich eine Vielzahl aktuell aktivierter Prozesse gegenseitig behindern und zu Dysregulationen führen. In der frühkindlichen Lebensphase formt sich die Persönlichkeitsstruktur des Menschen heraus, jedoch können Mangelerfahrungen noch nicht sprachlich kodiert werden. Solche defizitären Erfahrungen wirken im Unbewussten fort und sind für die Aufarbeitung schwer zugänglich. Das verletzte Selbst von sozial benachteiligten Menschen versucht sich zu schützen, indem es sich gegen äußere Zugriffe immunisiert und Vermeidungsstrategien entwickelt. Eine Vielzahl entwicklungs- und neuropsychologischer Studien bestätigen, dass sich die Störung emotionaler und sozialer Kompetenzen bereits in den ersten Lebensabschnitten strukturell niederschlagen und manifestieren.

\subsection{Bedürfnis nach Orientierung und Kontrolle}

Prinzipiell ist das Bedürfnis nach Orientierung und Kontrolle eng mit anderen Grundbedürfnissen assoziiert. Personen mit positiven Kontrollüberzeugungen

(c) Vandenhoeck \& Ruprecht GmbH \& Co. KG, Göttingen 2007 
verfügen über ein höheres $\mathrm{Maß}$ an Selbstwirksamkeitserwartungen, Selbstvertrauen und Lebenszufriedenheit. Dadurch fühlen sie sich insgesamt wohler und sind optimistischer und resistenter gegenüber Stress (Bandura, 1997). Verletzungen des Kontrollbedürfnisses münden in emotionsorientiertem Verhalten anstelle eines problemorientierten Bewältigungsverhaltens (Lazarus u. Folkman, 1984). Personen mit emotionsorientiertem Verhalten leiden häufiger unter körperlichen und psychischen Störungen, Stress, generalisierten Beeinträchtigungen des Wohlbefindens, Frustrationen, Ängsten, erlernter Hilflosigkeit und Depression (Flammer, 1990). Die Unkontrollierbarkeit aversiver Ereignisse in den ersten Lebensjahren ist einer der wichtigsten Vulnerabilitätsfaktoren für Erkrankungen im Erwachsenenalter und für hohe Komorbidität. Viele Verletzungen des Kontrollbedürfnisses in der frühen Kindheit, z. B. chronische Vernachlässigung tauchen später in posttraumatischen Belastungsstörungen wieder auf (Armbruster, 2000; Armbruster u. Bartels, 2005).

\subsection{Bedürfnis nach Bindung}

In den ersten Lebensmonaten sind Säuglinge vollkommen von ihren Bezugspersonen abhängig. Bereits in dieser Zeit verinnerlichen sie ihre frühen Beziehungserfahrungen, welche im weiteren Entwicklungsverlauf ihre Erwartungen an die eigene Selbstwirksamkeit (Bandura, 1997) und die Kontrolle über Ereignisse (Masten u. Coatsworth, 1998) bestimmen. Wenn Säuglinge und Kleinkinder die Erfahrung machen, dass bestimmte primäre Bezugspersonen stets dann verfügbar sind, wenn sie es brauchen, bahnen sich in ihnen positive Strukturen und sie sind in der Lage, alle kommenden Entwicklungsaufgaben besser zu bewältigen. So gelingt es ihnen im Kindesalter besser, soziale Kontakte aufzunehmen, und im Jugend- und Erwachsenenalter, stabilere intime Beziehungen zum anderen Geschlecht aufzubauen (Keller, 1997; Grossmann et al., 2002) und verhilft dauerhaft zu einer stabilen Erwartungshaltung. Auf der anderen Seite führen Zurückweisungen und fehlende Verlässlichkeit, die unsicher gebundene Kinder im Alter von wenigen Monaten erfahren, zu sehr heftigen Gefühlsregungen. Diese legen sich im impliziten Gedächtnis nieder und bahnen negative Wahrnehmungs-, Verhaltens-, emotionale und motivationale Reaktionsbereitschaften.

\subsection{Bedürfnis nach Selbstwerterhöhung}

Das Grundbedürfnis nach Selbstwerterhöhung setzt sich aus dem Bewusstsein seiner selbst und der Fähigkeit zu reflexivem Denken zusammen. In der kindlichen Entwicklung dauert es einige Jahre, bis sie sich so weit herausgebildet haben, dass Empfindungen der Selbstwertverletzung wie z. B. Scham oder Kränkung überhaupt als Kognition in Erscheinung treten. Im ersten Lebensabschnitt können zwar das Kontroll- und Bindungsbedürfnis frustriert werden, jedoch nicht das Bedürfnis nach Selbstwerterhöhung. Nichtsdestotrotz beeinflussen die von außen provozierte Nichtbefriedigung der Bedürfnisse auf der Kontroll- und Bindungsdimension

(C) Vandenhoeck \& Ruprecht GmbH \& Co. KG, Göttingen 2007 
das sich formierende Selbst in dieser frühen Zeit negativ und führen somit zum verinnerlichten negativen Selbstbild in der späteren Kindheit und im Jugendalter. Die wiederholten vorsprachlichen Frustrationen von Erwartungen münden in dominanten, dem Bewusstsein nicht zugänglichen Vermeidungsmustern, da sich die intentionalen Annährungsschemata nicht ausprägen konnten.

\subsection{Bedürfnis nach Lust}

Das Bedürfnis nach Lustgewinn und Unlustvermeidung geht automatisch mit positiv und negativ bewerteten Ereignissen einher. Diese rufen Annährungs- und Vermeidungstendenzen hervor. Kinder, die wiederholt unangenehme und frustrierende Erfahrungen erleben, unterdrücken diejenigen Verhaltensweisen, die mit Misserfolgen einhergehen. So verhindern sie einerseits auf Dauer negative Emotionen. Andererseits werden weniger Annährungsschemata durch neuronale Bahnung und Strukturbildung erzeugt. Kinder mit einem hohen Maß an Unlusterfahrungen sind störanfälliger. Sie beantworten Frustrationen ihres Bindungs- und Kontrollbedürfnisses mit negativen Emotionen und höherer Irritierbarkeit. In ihrem Explorations- und Interaktionsverhalten reagieren sie eher ausweichend. Diese ersten Lebenserfahrungen sind vorsprachlich, graben sich tief in das implizite Gedächtnis ein und sind später nur schwer zugänglich. Dennoch beeinflussen sie das Selbst in seinen Verhaltens- und Lernbereitschaften in der Zukunft nachhaltig, wodurch sich das Risiko von Entwicklungsrückständen, sozioemotionalen Störungen und kognitiven Defiziten erhöht (Brähler, Schumacher, Strauß, 2002).

\subsection{Reflexion}

Dennoch stellen diese Befunde kein Anlass zu Hoffnungs- und Tatenlosigkeit dar. Bei Vorliegen negativer Bindungserfahrungen ist es durchaus möglich durch gezielte Maßnahmen die elterliche Empathie zu verbessern (van den Boom, 1994). Außerdem gehen Epstein und Morling (1995) davon aus, dass Personen trotz ausgeprägter Vermeidungsstrategien ein Bedürfnis nach selbst aufwertenden Wahrnehmungen haben. Ihren Befunden zufolge tendiert das implizite Funktionssystem eher zu selbstwerterhöhenden Emotionen und Verhaltensweisen als das explizite. Es liegt demzufolge nahe, Menschen mit schlechtem Selbstwertgefühl auf implizitem Weg positiv zu beeinflussen. So spielt der implizite Funktionsmodus (Grawe, 1998, 2004) im Zusammenhang mit dem Lernen in der ELTERN-AG eine große Rolle.

\section{Empowerment}

Erhalten Menschen, die unter risikoreichen Bedingungen leben, positiv bewertete Einordnungen, so ergeben sich im Sinne eines reziproken Determinismus erstaunliche Veränderungen. Sie trauen sich einerseits mehr zu und werden aktiver. Auf der anderen 
Seite werden sie auch von ihrer Umwelt positiver wahrgenommen. Diese positiven Erwartungen der Umwelt führen wiederum zu einem neuen bejahenden Lebensgefühl.

Der ELTERN-AG gelingen diese positiven Zuschreibungen durch die stringente Anwendung von Empowerment. Im Allgemeinen umfasst Empowerment das Ziel, für Menschen die Möglichkeiten zu erweitern, ihr Leben zu bestimmen (Rappaport, 1985). Im Sinne der ELTERN-AG beinhaltet Empowerment die Deklaration der Eltern als Experten ihrer eigenen Lebensgeschichte, dadurch wird ihnen die Macht über ihre eigenen Lebensverhältnisse zurückgegeben. Von zentraler Bedeutung ist einerseits die Einwirkung auf andere (to empower people) und andererseits die Einwirkung auf sich selbst (self-empowerment). Ein wichtiges Mittel dabei sind die Selbstbemächtigung und die Selbstaneignung von Lebenskräften (Herriger, 1997), die in der ELTERN-AG durch spezifische Formen der Kommunikation und Interaktion realisiert werden. Praktisch gesehen, bedeutet das, dass die Ressourcen und Potentiale der Teilnehmer zur Selbsthilfe bei jeder sich bietenden Gelegenheit von den Mentoren aufgespürt und unermüdlich sichtbar gemacht werden. In den Treffen fokussieren die Mentoren, die den Eltern ohne hierarchischen Abstand und auf gleicher Augenhöhe begegnen, die positiven Anteile, d. h. Stärken, Begabungen und soziale Fähigkeiten des Einzelnen werden besonders betont. Von hoher sozialer Relevanz ist ebenfalls die Reflexion in der Gruppe. Sie vermittelt die Erfahrung des sozialen Integriertseins und legt damit den Grundstein für den Aufbau sozialer Netzwerke.

\section{Das Konzept der ELTERN-AG}

Konventionelle Programme zur Steigerung der elterlichen Erziehungskompetenz gibt es zur genüge, doch Eltern mit langjährigen Misserfolgserfahrungen, gescheiterten Bildungsbiographien, denen ein Platz am Arbeitsmarkt versperrt bleibt, nehmen diese Maßnahmen selten für sich in Anspruch.

Die ELTERN-AG stellt in punkto Erreichbarkeit dieser Eltern im deutschsprachigen Raum eine Ausnahme dar. Wenngleich das Trainingsprogramm grundsätzlich für Eltern aus allen Bevölkerungsschichten anwendbar ist, gilt das besondere Augenmerk jenen gesellschaftlichen Randgruppen, die oft als Bildungsferne, Modernisierungsverlierer, von Armut oder Migration Betroffene oder Menschen in besonderen Lebenslagen umschrieben werden. Es orientiert sich an zwei Kerndimensionen:

- der Schulung der Früherziehungskompetenzen von Eltern (z.B. Papoušek, 1996) und

- der Stärkung der Selbsthilfepotentiale der Eltern durch Empowerment (Armbruster, 2006).

Mit Früherziehungskompetenzen ist im Wesentlichen ein hoher Grad in den Fähigkeiten zu liebevoller Zuwendung, Wahrnehmung, Respekt, Lenkung und Förderung sowie der positiven Verstärkung des erwünschten Verhaltens gemeint. Durch Em-

(C) Vandenhoeck \& Ruprecht GmbH \& Co. KG, Göttingen 2007 
powerment lernen die Eltern positive Aspekte zu verstärken, sich selbst zu motivieren und sich mit anderen zusammen zu tun, um einander gegenseitig Unterstützung zu geben. Die Ausprägung dieser Fähigkeiten wird maßgeblich durch das Training der ELTERN-AG beeinflusst und gesteigert. Durch das Training der ELTERN-AG lernen die Eltern, die unterschiedlichen kindlichen Entwicklungsschritte zu begleiten und zu fördern. Beide Dimensionen - Elternkompetenzen und Empowerment - ergänzen und verstärken sich gegenseitig.

Das Präventionsprogramm ELTERN-AG wendet sich primär an Menschen in der Familienplanungsphase und junge Eltern mit Kindern im Vorschulalter. Die ELTERN-AG wird von so genannten „Mentoren“ durchgeführt. Die MentorInnen sind keine Erziehungsberater im traditionellen Sinn, sondern Befähiger, Begleiter und Ermöglicher! Wichtigster Grundsatz ist die „gleiche Augenhöhe“ und die „Mobilisierung der Ressourcen“ der Kursteilnehmer. Die ELTERN-AG ist geschlechtsgerecht ausgerichtet und genderorientiert. Die Eltern sind im Setting der ELTERNAG von dem Druck entlastet, perfekte Pädagogen sein zu müssen. Sie diskutieren und erarbeiten in der Gruppe der ELTERN-AG Lösungsvorschläge, die sie zunächst probehandelnd mit anderen Gruppenmitgliedern ausprobieren und später auf ihre eigene Familie übertragen. Die Eltern vergrößern ihr Wissen über die kindliche Entwicklung und lernen die Grundlagen eines effektiven Erziehungsverhaltens kennen. Ausstattung, Umfang, Inhalte und Durchführung der Kurse sind einer kontinuierlichen Evaluation unterworfen.

Dem Prinzip der Niedrigschwelligkeit räumt die ELTERN-AG in der praktischen Umsetzung hohe Priorität ein. Konzeptionell wird Niedrigschwelligkeit durch das Prinzip der „gleichen Augenhöhe“, d. h. durch den Verzicht der MentorInnen auf den Expertenstatus, umgesetzt. Sie arbeiten als Befähiger, die die Eltern als „Experten ihrer Kinder" ausdrücklich wertschätzen. Ihre zugrunde liegenden Ressourcen werden zugänglich gemacht. Ein weiteres wichtiges Kennzeichen der Niedrigschwelligkeit ist die "Selbsthilfeorientierung". Die Eltern übernehmen mit zunehmender Kursdauer sukzessive selbst die Regie der ELTERN-AG und bilden vernetzte Selbsthilfezirkel innerhalb eines Netzwerkes. Die MentorInnen lernen in ihrer Ausbildung, von Beginn an alle Teilnehmer aktiv in den Ablauf der Sitzungen, in die Gespräche, Übungen und Diskussionen, mit einzubeziehen. Die begleitenden Trainingsmaterialien sind durch klare Sprache und anschauliche Illustration so gestaltet, dass auch wenig Lesegeübte und des Lesens Unkundige die didaktischen Materialien mit Gewinn benutzen können.

Die Effekte der ELTERN-AG verteilen sich auf folgende Hauptgebiete:

1. Für sozial benachteiligte Kinder erhöht sich die Chance beträchtlich, zu einem biographisch sehr frühen Zeitpunkt eine kompetente Erziehung, Stimulation und Förderung durch verantwortungsbewusste und sensibilisierte Eltern zu erfahren, die im Austausch mit anderen Eltern stehen. Dadurch verbessern sich 
ihre Chancen auf eine gute psychosoziale Entwicklung und auf Schul- und Ausbildungserfolg.

2. Eltern, vor allem solche, die den traditionellen Unterstützungsangeboten eher skeptisch bis ablehnend gegenüberstehen $-z$. B. so genannte Bildungsferne und Modernisierungsverlierer - werden in der Phase der Nestbildung und der frühen Kindheit als erste und entscheidende Erziehungs- und Bildungsinstanz aufgewertet und gestärkt. Sie lernen, ihre Kompetenzen wahrzunehmen, zu nutzen und auszuweiten.

3. Durch das Empowerment lernen die Eltern ihre eigenen Kräfte, Fähigkeiten und Potentiale auszuschöpfen und sich in ELTERN-AG-Selbsthilfegruppen gegenseitig zu unterstützen.

\section{Aufbau der ELTERN-AG}

Vorlaufphase. In der Vorlaufphase, die insgesamt ca. sechs Wochen in Anspruch nimmt, führen die zwei MentorInnen zunächst Gespräche mit relevanten sozialen Institutionen und wichtigen Multiplikatoren im Stadtteil oder in der Region wie Jugendämtern, Kirchengemeinden, Kindergärten und Schulen, Kinderärzten, Hebammen, ErzieherInnen etc, welche die lokalen Bevölkerungsgruppen und Umgebungsbedingungen am besten kennen. Sie werden mit dem ELTERN-AG-Programm vertraut gemacht und um Kooperation gebeten. In der Regel treten diese als Veranstalter auf, in deren Räumen die Kurse stattfinden.

Randständige Personen sind i.d.R. schwer für Bildungsmaßnahmen und Elternkurse zu gewinnen. Daher ist eine zugehende und aufsuchende Arbeitsweise zentral. Die MentorInnen sprechen in einem zweiten Schritt der Elternwerbung in ausgesuchten Stadteilen und Treffpunkten die Eltern für die ELTERN-AG an, also in der konkreten Lebenswelt, dort, wo sie ihre Adressaten am ehesten finden, wodurch sich die Zugangsbarrieren verringern. Sie machen durch originelle, lustige Aktionen etwa auf Spielplätzen oder vor Kindergärten auf sich aufmerksam und stellen das Programm auf eine einladende Art vor, die die Neugier weckt und den Eltern Lust zur Teilnahme macht. Alle Eltern erhalten die Information, dass die Teilnahme an der ELTERN-AG kostenlos ist und unter Vertrauensschutz steht.

Sind zehn Eltern "geworben“, welche die Kriterien der Zielgruppe, nämlich Bildungsferne, soziale Benachteiligung oder Migrationshintergrund erfüllen, kann sich die ELTERN-AG-Gruppe konstituieren. Mit ihr besprechen die Mentoren, wo die Treffen stattfinden sollen. Lange Anfahrtswege und fremde Umgebungen werden vermieden, da sie mit einem hohen Zeitaufwand verbunden sind bzw. Angst machen. Zu jedem Treffen wird Kinderbetreuung angeboten.

Initial- und Konsolidierungsphase. Die ELTERN-AG umfasst stets die Initial- und Konsolidierungsphase mit insgesamt 20 Sitzungen, die sich aus je zwei Gruppenstunden à 45 Minuten zusammensetzen. Jede ELTERN-AG wird von zwei Mentoren 
vorbereitet und geleitet. Im Sinne des geschlechtergerechten Gender-Ansatzes erfolgt die Anleitung nach Möglichkeit jeweils durch eine Frau und einen Mann. Jede ELTERN-AG-Sitzung wird unter Berücksichtigung von Datenschutzgesichtspunkten durch die Mentoren standardisiert, dokumentiert und evaluiert.

Die ELTERN-AG startet mit der Initialphase, in der die ersten zehn Sitzungen stattfinden. Ihr Schwerpunkt liegt in der Herausbildung von geregelten Gruppenabläufen, der Bearbeitung der Erziehungs- und Gruppenregeln sowie der Förderung einer Gruppenidentität. Die Inhalte der Treffen orientieren sich an den Interessen und den Bedürfnissen der teilnehmenden Eltern. Die Mentoren übernehmen die Rolle von Begleitern und Beratern auf gleicher Augenhöhe, welche den Austausch zwischen den Eltern anregen. In der Initialphase sind die Eltern jedoch noch mehr als in der Konsolidierungsphase auf die „Regieanweisungen“ der Mentorinnen und Mentoren angewiesen: Diese kennen den Rahmen und die Struktur des dreigliedrigen Ablaufes der ELTERN-AG.

Die Konsolidierungsphase erstreckt sich über die Sitzungen 11 bis 20. Sie bietet bei gleicher Struktur den Eltern die Möglichkeit, die Gestaltung der Treffen mehr in die Hand zu nehmen, indem sie ihre Erfahrungen aus den ersten zehn ELTERNAG-Sitzungen selbst anwenden und verstärkt Verantwortung für die Gruppe übernehmen. Damit werden die Eltern vorbereitet, die Gruppe selbständig nach den 20 Sitzungen weiterzuführen.

Nach den 20 ELTERN-AG-Treffen wird ein Abschlussbericht mit den folgenden Punkten erstellt: Ort, Zeitraum, Teilnehmerzahl, Ort und Art der Elternwerbung, vermittelte fachliche Inhalte, Verlauf, Ergebnisse, Reflexion und Empfehlungen, Zufriedenheit der Eltern, Hinweise in Bezug auf Nachhaltigkeit, Vernetzungsaspekte und Anschlussaktivitäten an die Maßnahme.

\subsection{Die drei Kernelemente der ELTERN-AG}

Die Struktur der drei Kernelemente der ELTERN-AG gründen auf den Erkenntnissen des impliziten Lernens. Die Eltern werden eingeladen, sich einzubringen und mitzugestalten. Die „seminaristischen Teile“ sind auf wenige Minuten beschränkt, um zu verhindern, dass durch den „Schulcharakter der Informationsvermittlung “ bei den Eltern zu viele negative Assoziationen und Ängste vor Überforderung entstehen und sie in den „Vermeidungsmodus“ überwechseln. Aber auch wenn aus konzeptionellen Gründen kein „deklaratives Lernen“ eingebaut ist, erhalten die Eltern auf mehreren Kanälen (z. B. visuell, akustisch, motorisch) und Ebenen (z. B. Learning by doing, Modelllernen, sozialer Vergleich) vielfältige Informationen, die zu einer Erweiterung und Umstrukturierung vorhandener kognitiver Muster führen.

Jede Sitzung der ELTERN-AG ist von einer dreigliedrigen Struktur getragen. Für jedes der drei Kernelemente sind ungefähr 30 Minuten Zeit vorgesehen.

- Kognition: „Schlaue Eltern“

- Stressmanagement: „Relax“

- Soziales Lernen in der Gruppe: „Mein aufregender Elternalltag“

(C) Vandenhoeck \& Ruprecht GmbH \& Co. KG, Göttingen 2007 
Schlaue Eltern. Den „Türaufmachern“ Begrüßung und Warming-up schließt sich das erste Kernelement, der Teil Schlaue Eltern, an. In diesem auf Wissenszuwachs ausgerichteten Teil haben die Eltern die Gelegenheit, sich genau diejenigen Themen (z. B. Sauberkeitserziehung, Trotzalter, Meilensteine der Entwicklung) rund um die kindliche Entwicklung zu wünschen, die ihnen unter den Nägeln brennen. Die Mentoren bereiten für die nächste Sitzung eine leicht verständliche Darstellung des aktuellen Wissensstandes mittels eines ca. zehnminütigen Kurzvortrages vor. Sie fassen ihre Erkenntnisse in einfachen Worten zusammen. In den folgenden 20 Minuten diskutieren die Eltern das Gehörte und bringen ihre eigenen Erfahrungen zu diesem Thema ein.

Die Mentoren arbeiten darauf hin, die Eltern durch Aktivierung an die „Selbsterschließung" von Wissen heranzuführen. Im Sinne Positiver Psychologie und der Ressourcenorientierung werden alle Beiträge wohlwollend verstärkt. Durch die Kombination von Wissensvermittlung und anschließender Diskussion fühlen sich die Eltern bereichert und zugleich als „Experten“ ermutigt. Das gesteigerte persönliche Engagement erhöht die Aufnahmebereitschaft der Eltern und fördert ihr Selbstbewusstsein.

Relax. Der Alltag führt bei vielen sozial benachteiligten Eltern zu Dauerstress und einem konstant überhöhten Erregungsniveau. Sie treffen in ihrer Umgebung i.d.R. auf keine guten Modelle, die ihnen zeigen könnten, wie man sich entspannt und wieder Ordnung in das Gefühlschaos bringt. Ohne es zu wollen, empfinden sie dann manchmal sogar die eigenen Kinder als große Belastung und schieben ihnen die Schuld für den Stress zu. Die Kinder als schwächstes Glied in der Kette werden leicht zu Empfängern der negativen Emotionen.

Im Relax-Teil lernen die Eltern erst einmal die eigene Anspannung wahrzunehmen und über sie zu sprechen. Sie erkennen, an welchen Stellen im Alltag es am ehesten Stress mit ihren Kindern gibt. Die Mentoren lenken die Aufmerksamkeit der Eltern auf ihre „Bauchgefühle“, wodurch sie ein erweitertes und sensibleres Körperbewusstsein erlernen. So haben sie eher die Chance, stressige Situationen „herunterzuregulieren" und Techniken für ein konstruktives Stressmanagement zu entwickeln.

In den ersten 15 Minuten reden die Eltern darüber, welche Situationen sie aus ihrem Alltag kennen, in welchen sie oft das Gefühl haben, „Hilfe, ich ertrage mein Kind nicht mehr!" Sie tauschen sich über Stressreaktionen und Lösungsmöglichkeiten aus, die sie in solchen Situationen ausprobiert haben. Gemeinsam schätzen sie in der Gruppe die Wirksamkeit ihres Vorgehens ein. Die Eltern verraten sich untereinander ihre ganz persönlichen „Tipps und Tricks" und diskutieren, was bei ihnen gut klappt.

In den zweiten 15 Minuten laden die Mentoren dazu ein, die „Tipps und Tricks“ auszuprobieren, um die Wirkung „am eigenen Leib“ zu erfahren. Sie greifen die Erfahrungen der einzelnen Teilnehmer auf und bringen selbst Anregungen und Entspannungsübungen ein. Die Eltern können so herausfinden, was für sie nützlich ist, wenn sie sich gestresst fühlen. Manche Übungen können sie zusammen mit ihren Kindern machen, um sich mit ihnen zu entspannen und wieder einen positiveren Kontakt mit ihnen zu bekommen. Die Eltern lernen, sich Inseln in den Tag einzubauen, auf denen sie sich erholen können. 
Mein aufregender Elternalltag. Das Kernelement „Mein aufregender Elternalltag“ lässt den Eltern weitgehend freie Hand. Sie können spontan Ereignisse aus der letzen Woche berichten oder die Zeit dazu nutzen, um ein ganz bestimmtes Erziehungsproblem vertieft zu erörtern. Hier berichten sie, was sie in der vergangenen Woche mit ihren Kindern erlebt haben, welche schönen, aber auch welche schwierigen Situationen es gab. Sie erzählen, was für sie aus der letzten ELTERN-AG nützlich war und was sie davon in ihren Alltag eingebaut haben.

Die Eltern erfahren in der Gruppe, dass die Anderen auch „nur mit Wasser kochen“. Sie erfahren durch die Gruppe, dass sie mit ihren Sorgen und Problemen nicht allein sind und erleben, dass es sich lohnt, auch mal jemand anderes zu fragen und sich gegenseitig zu unterstützen. Mit dem „aufregenden Elternalltag“ haben die Eltern ein Forum, auf welchem sie von anderen Verhaltensweisen hören, diese diskutieren, ausprobieren und einüben können. Auch das Besprechen von „Hausaufgaben" vom letzten Treffen findet in diesem Abschnitt statt.

Im Sinne des Empowerment-Ansatzes lernen die Eltern in diesem Teil, sich und Andere kennen. Die Mentoren achten darauf, die Bemühungen der Eltern angemessen zu würdigen und sie in der Überzeugung, richtig handeln und erziehen zu können, liebenswert zu sein und gut für sich und die Familie zu sorgen, zu stärken. Sie konnotieren alle Anstrengungen der Eltern um eine bessere Erziehung positiv. Die Mentoren unterstützten einen Prozess, welcher zum Ziel hat, das Bewusstsein der Eltern bzgl. ihrer Problemlösefähigkeiten zu heben und auf andere Ebenen des Erziehungsalltags, z.B. im Austausch mit Erzieherinnen der KiTa, zu transferieren.

\section{Erste Ergebnisse}

In einem Zeitraum von April 2004 bis Dezember 2006 erfolgten in ganz SachsenAnhalt 37 ELTERN-AG-Gruppen. 70 Mentoren wurden ausgebildet. 323 Eltern, davon 84,8 \% weiblichen Geschlechts und 15,2 \% männlichen Geschlechts, die den soziodemographischen Kriterien entsprachen, und über 600 Kinder haben in dieser Zeit von der ELTERN-AG profitieren können. Im Verlauf der 20 Sitzungen lag die durchschnittliche Anwesenheit bei 70,3\%, wobei lediglich knapp $10 \%$ der Teilnehmer ohne Grund den Kurs abgebrochen haben. 11 \% der Eltern konnten den Sitzungen nicht mehr inne wohnen, da sie schwerwiegende persönliche Probleme hatten, unter anderem Trennung vom Partner, erneute Schwangerschaft, Erkrankung des Kindes. 3,9\% der Eltern haben aufgrund einer Maßnahme des Arbeitsamtes zeitlich nicht mehr an dem Kurs teilnehmen können. 3,4 \% der Teilnehmer sind schwer erkrankt, dadurch bestand für sie keine Möglichkeit mehr, an den Treffen teilzunehmen. Die Kurse fanden in 16 verschiedenen Orten (Blankenburg, Burg, Dessau, Genthin, Halberstadt, Haldensleben, Magdeburg, Osterburg, Parey, Quedlinburg, Schönebeck, Seehausen, Staßfurt, Stendal, Wernigerode, Wittenberg) statt. Eine 
Vielzahl der ELTERN-AGs trifft sich nach Beendigung des Kurses als Selbsthilfegruppe mit Patenschaft der bisherigen Mentoren weiter. 89,4 \% ( $\mathrm{n}=198)$ der Eltern waren mit den Themen, die in der ELTERN-AG angesprochen wurden, zufrieden bzw. sehr zufrieden.

Tab. 2: Zufriedenheit mit den angesprochenen Themen in der ELTERN-AG

\begin{tabular}{lcc}
\hline & Häufigkeit & Prozent \\
\hline sehr unzufrieden & 12 & 6,1 \\
unzufrieden & 1 & 0,5 \\
mittelmäßig & 6 & 3,0 \\
zufrieden & 56 & 28,3 \\
sehr zufrieden & 121 & 61,1 \\
keine Angaben & 2 & 1,0 \\
gesamt & 198 & 100,0 \\
\hline
\end{tabular}

Im Verlaufe des Kurses hatten 70,2 \% der Teilnehmer die Möglichkeit, ihre Erfahrungen im Alltag oft bis sehr oft anzuwenden. 24,7 \% der Eltern bemerkten bei der Umsetzung in ihrer häuslichen Umgebung Schwierigkeiten. So gelang es ihnen bisher nur manchmal die neu erlernten Fähigkeiten umzusetzen. Diese Personen wünschten sich nach Beendigung des Kurses, an einem weiteren teilnehmen zu dürfen.

Tab. 3: Werden Erfahrungen der ELTERN-AG in Zukunft genutzt?

\begin{tabular}{lcc}
\hline & Häufigkeit & Prozent \\
\hline nie & 2 & 1,0 \\
selten & 3 & 1,5 \\
manchmal & 25 & 12,6 \\
oft & 95 & 48,0 \\
sehr oft & 68 & 34,3 \\
keine Angaben & 5 & 2,5 \\
gesamt & 198 & 100,0 \\
\hline
\end{tabular}

Insgesamt hatten 82,3\% der teilnehmenden Personen nach Beendigung des Kurses das Gefühl, ihre Erfahrungen auch weiterhin oft bis sehr oft nutzen zu können. Sie fühlten sich gestärkt im Umgang mit ihren Kindern, schätzten insgesamt die Beziehung zu ihren Kindern positiver ein und fühlten sich sicherer im Umgang mit Problemen. Des Weiteren schätzten sie den Austausch mit anderen Eltern. Der Wandel, den die Eltern während des Kurses an sich wahrnahmen, fiel den Mentoren auf und wurde als positive Veränderung von den Multiplikatoren bestätigt. Alle Eltern hatten mehr oder weniger ein soziales Netzwerk aufbauen können, sei es durch das Fortbestehen der Treffen über das offizielle Ende des Kurses hinaus (74,25\%) oder

(C) Vandenhoeck \& Ruprecht GmbH \& Co. KG, Göttingen 2007 
durch die positivere Kommunikation verbunden mit einer besseren Zusammenarbeit mit den Multiplikatoren der frühpädagogischen Einrichtungen (24,75 \%). $1 \%$ der Teilnehmer haben hierzu keine Angaben getätigt.

Tab. 4: $\quad$ Transfer in den Alltag

\begin{tabular}{lcc}
\hline & Häufigkeit & Prozent \\
\hline nie & 4 & 2,0 \\
selten & 3 & 1,5 \\
manchmal & 49 & 24,7 \\
oft & 84 & 42,4 \\
Sehr oft & 55 & 27,8 \\
keine Angaben & 3 & 1,5 \\
gesamt & 198 & 100,0 \\
\hline
\end{tabular}

Wie gestärkt und positiv motiviert sich die Eltern zum Ende des Kurses fühlten, sowohl im Umgang mit der eigenen Kompetenz, dem eigenen Stressverhalten und im Umgang mit ihren Kindern, zeigt sich letzten Endes auch darin, dass $97 \%$ der Teilnehmer, die ELTERN-AG weiterempfahlen.

\section{Literatur}

Armbruster, M. (Hrsg.) (2000). Misshandeltes Kind. Hilfe durch Kooperation. Freiburg: Lambertus.

Armbruster, M., Bartels, V. (2005). Kooperation der verschiedensten Dienste bei Kindesmisshandlung, -vernachlässigung, sexuellem Missbrauch und Vernachlässigung. In G. Deegener, W. Körner (Hrsg.), Kindesmisshandlung und Vernachlässigung. Göttingen: Hogrefe.

Armbruster, M. (Hrsg.) (2006). Eltern-AG. Das Empowerment-Programm für mehr Elternkompetenz in Problemfamilien. Heidelberg: Carl-Auer.

Bandura, A. (1997). Self-efficacy in changing societies. Cambridge: Univ. Press.

Baumert, J. (Hrsg.) (2001). PISA 2000. Basiskompetenzen von Schülerinnen und Schülern im internationalen Vergleich. Opladen: Leske \& Budrich.

Bos, W. et al. (2003). Heterogenität. Eine Herausforderung an die empirische Bildungsforschung. Münster: Waxmann.

Brähler, E., Schumacher, J., Strauß, B. (2002). Diagnostische Verfahren in der Psychotherapie. Göttingen: Hogrefe.

Braun, A. K. (2006). Wie Gehirne Lernen lernen. Oder: Früh übt sich, wer ein Meister werden will. Magdeburger Akademie für Praxisorientierte Psychologie e. V., Vortrag vom 02.02.06, Magdeburg.

Epstein, S. (1990). Cognitive-experiental self-theory. In L. A. Pervin (Hrsg.), Handbook of personality. Theory an research (S. 165-192). New York: Guil.

Epstein, S. (1993). Implications of cognitive-experiential self-theory for personality and developmental psychology. In D. C. Funder, R. D. Parke, C. Tomlinson-Keasey, K. Widaman 
(Hrsg.). Studying lives through time: Personality and development. Washington DC. American Psychological Association, S. 399-438.

Flammer, A. (1990). Erfahrungen der eigenen Wirksamkeit. Einführung in die Psychologie der Kontrollmeinung. Bern: Huber.

Fthenakis, W. (2003). Auf den Anfang kommt es an! - Perspekive zur Weiterentwicklung des Systems der Tageseinrichtungen für Kinder in Deutschland. Weinheim: Beltz.

Grawe, K. (1998). Psychologische Therapie. Göttingen: Hogrefe.

Grawe, K. (2004). Neuropsychotherapie. Göttingen: Hogrefe.

Grossmann, K. E., Grossmann, K., Winter, M., Zimmermann, P. (2002). Bindungsbeziehungen und Bewertung von Partnerschaft. Von früher Erfahrung feinfühliger Unterstützung zu späterer Partnerschaftsrepräsentation. In K. H. Brisch, K. E. Grossmann, K. Grossmann, L. Köhler (Hrsg.): Bindung und seelische Entwicklungswege. Grundlagen, Prävention und klinische Praxis (S. 125-164). Stuttgart: Klett-Cotta.

Herriger, N. (1997). Empowerment in der sozialen Arbeit. Eine Einführung. Stuttgart: Kohlhammer.

Keller, H. (1997). Entwicklungspsychopathologie. Das Entstehen von Verhaltensproblem in der frühesten Kindheit (S. 624-642). In H. Keller (Hrsg.), Handbuch der Kleinkindforschung. Bern: Huber.

Krumm, V.,Wetzek, G., Tietze, W., Hundertmark-Mayser, J., Rossbach, H.-G., Paiacios, J. an Lerra, M. J. (1999). European Child Care and Education Study. School-age Assessment of Child Development. Long-term impact of Pre-school Experiences on School Success, and Family-School Relationships. Brüssel. Final Report for Work Package 2.

Lazarus, R. S., Folkman, S. (1984). Stress, appraisal, and coping. New York: Springer.

Masten, A. S., Coatsworth, J. D. (1998). The development of competence in favorable and unfavorable environments: Lessons from research on successful children. American Psychologist, 53, 205-220.

van den Boom, D. C. (1994). The influence of temperament an mothering on attachment and exploration. An experimental manipulation of sensitive responsivness among lower-class mothers with irritable infants. Child Development, 65,1457-1477.

Papoušek, M. (1996). Frühe Störungen der Eltern-Kind-Beziehung im Säuglingsalter. Neue Erkenntnisse der Bindungsforschung. In: Liga für das Kind, 27-47.

Rappaport, J. (1985). Ein Plädoyer für die Widersprüchlichkeit: Ein sozialpolitisches Konzept des „empowerment“ anstelle präventiver Ansätze. Verhaltenstherapie und psychosoziale Praxis, 2, 257-278.

Korrespondenzadresse: Korrespondenzadresse: Diana Sodtke, Hochschule Magdeburg-Stendal (FH), Breitscheidstr. 2, 39114 Magdeburg.

E-Mail: Diana.Sodtke@hs-magdeburg.de, Meinrad.Armbruster@hs-magdeburg.de 